\title{
Epstein-Barr virus-positive gastric cancer: a distinct molecular subtype of the disease?
}

\author{
Alexandre Andrade dos Anjos Jácome ${ }^{[1]}$, Enaldo Melo de Lima ${ }^{[1]}$, Ana Izabela Kazzi ${ }^{[1]}$, \\ Gabriela Freitas Chaves ${ }^{[1]}$, Diego Cavalheiro de Mendonça $a^{[1]}$, Marina Mara Maciel ${ }^{[1]}$ \\ and José Sebastião dos Santos ${ }^{[2]}$
}

[1]. Departamento de Oncologia Clínica, Hospital Mater Dei, Belo Horizonte, Minas Gerais, Brasil. [2]. Departamento de Cirurgia e Anatomia, , Faculdade de Medicina de Ribeirão Preto, Universidade de São Paulo, Ribeirão Preto, São Paulo, Brasil.

\begin{abstract}
Approximately $90 \%$ of the world population is infected by Epstein-Barr virus (EBV). Usually, it infects B lymphocytes, predisposing them to malignant transformation. Infection of epithelial cells occurs rarely, and it is estimated that about to $10 \%$ of gastric cancer patients harbor EBV in their malignant cells. Given that gastric cancer is the third leading cause of cancerrelated mortality worldwide, with a global annual incidence of over 950,000 cases, EBV-positive gastric cancer is the largest group of EBV-associated malignancies. Based on gene expression profile studies, gastric cancer was recently categorized into four subtypes; EBV-positive, microsatellite unstable, genomically stable and chromosomal instability. Together with previous studies, this report provided a more detailed molecular characterization of gastric cancer, demonstrating that EBV-positive gastric cancer is a distinct molecular subtype of the disease, with unique genetic and epigenetic abnormalities, reflected in a specific phenotype. The recognition of characteristic molecular alterations in gastric cancer allows the identification of molecular pathways involved in cell proliferation and survival, with the potential to identify therapeutic targets. These findings highlight the enormous heterogeneity of gastric cancer, and the complex interplay between genetic and epigenetic alterations in the disease, and provide a roadmap to implementation of genome-guided personalized therapy in gastric cancer. The present review discusses the initial studies describing EBV-positive gastric cancer as a distinct clinical entity, presents recently described genetic and epigenetic alterations, and considers potential therapeutic insights derived from the recognition of this new molecular subtype of gastric adenocarcinoma.
\end{abstract}

Keywords: Stomach neoplasms. Epstein-Barr virus infections. Genomics. Epigenomics. Molecular targeted therapy.

\section{INTRODUCTION}

Since its first description in 1964, Epstein-Barr virus (EBV), a $\gamma$-herpes virus, has been closely related to the pathogenesis of malignancies, mainly lymphoids ${ }^{(1)}$. EBV preferentially infects B lymphocytes, in which it tends to persist as a latent, asymptomatic infection and, by expression of proteins that influence the host cell cycle, it predisposes to malignant transformation ${ }^{(2)}$. EBV infection associated with malignancy was first described in endemic Burkitt's lymphoma; however, it has since been consistently identified as involved in the pathogenesis of Hodgkin's lymphoma and post-transplantation lymphomas ${ }^{(2)}$.

While the involvement of EBV in lymphoid malignancies is well described, the effects of EBV infection of other cell types, particularly epithelial cells, remain poorly understood. The role of EBV in the pathogenesis of nasopharyngeal carcinoma and in a subgroup of patients with gastric adenocarcinoma is recognized; however, the genetic and epigenetic alterations

Corresponding author: Dr. Alexandre Andrade dos Anjos Jácome.

e-mail: jacome@usp.br

Received 31 July 2015

Accepted 12 February 2016 responsible for these malignant phenotypes require further elucidation.

Given the global annual incidence of $>950,000$ cases of gastric adenocarcinoma ${ }^{(3)}$, and the known association of EBV infection with $8 \%-10 \%$ of cases $^{(4)}(5)$, including $80 \%$ of rare lymphoepithelioma-like gastric carcinoma ${ }^{(6)}(7)$ and $35 \%$ of stump gastric carcinomas ${ }^{(8)(9)}$, EBV-positive gastric cancer cases constitute the largest group of EBV-associated malignancies.

Gastric cancer (GC) is the third most common cause of cancerrelated mortality worldwide ${ }^{(3)}$. Approximately $95 \%$ of stomach neoplasms are adenocarcinomas ${ }^{(10)}$, and in this review we use these terms interchangeably. GC incidence is closely related to environmental factors, which is reflected in a characteristic geographic distribution, with Eastern countries, Eastern Europe and Latin America representing the greatest areas of risk for disease $^{(3)}$. GC has a dismal prognosis, with high relapse rates, even for localized tumors, and estimated survival times of only $8-10$ months in patients with recurrent or metastatic disease $\mathrm{e}^{(11)(12)}$.

Recognition of the heterogeneity of malignancies and elucidation of the detailed molecular biology of disease subtypes, both of which have allowed the identification of driver mutations as potential therapeutic targets, have significantly improved systemic cancer therapy (Figure 1). In addition, 


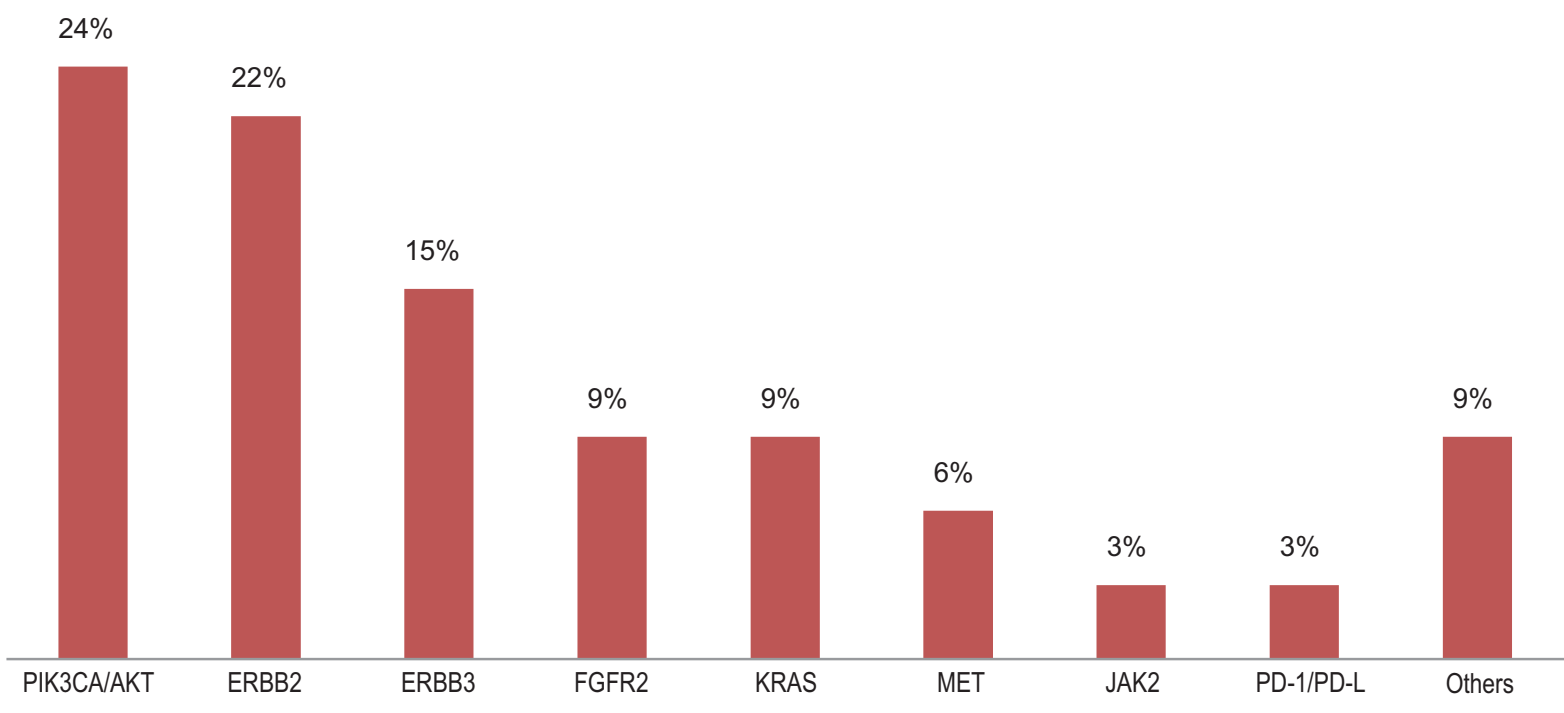

FIGURE 1 - Proportion of genetic abnormalities in gastric cancer.

the advent of monoclonal antibodies and tyrosine kinase inhibitors targeting key proteins in tumor proliferation have altered the landscape of systemic cancer therapy in recent years.

The ToGA study ${ }^{(13)}$ demonstrated that approximately $20 \%$ of GC patients have c-erbB2 amplification and/or HER2 protein overexpression, a receptor tyrosine kinase member of the epidermal growth factor receptor (EGFR) superfamily ${ }^{(13)}$ (14), providing a first step towards individualization of therapy. The study found that the addition of trastuzumab, a monoclonal antibody that inhibits HER2, to a standard chemotherapeutic regimen significantly improved overall survival ${ }^{(13)}$.

Through gene expression profile studies, a new molecular characterization of gastric adenocarcinoma has recently been proposed. The Cancer Genome Atlas (TCGA) Research Network has identified four distinct molecular subtypes of the disease, and one of these was characterized by positivity for EBV infection ${ }^{(15)}$. These data reinforce previous studies demonstrating that EBV infection induces unique genetic and epigenetic alterations in gastric epithelial cells, resulting in a distinct disease phenotype, and corroborate the hypothesis that EBV-positive GC is a distinct clinical entity ${ }^{(16)(17)(18)(19)}$. Molecular alterations unique to EBVpositive $\mathrm{GC}$ will enable identification of novel driver mutations and potential therapeutic targets.

This review aimed to revisit the initial studies describing EBV-positive GC as a distinct clinical entity, describe recently identified genetic and epigenetic alterations, and consider the potential therapeutic implications of the recognition of this new molecular subtype of gastric adenocarcinoma.

\section{Clinicopathological characteristics of gastric cancer}

World Health Organization (WHO) classification divides GC into four distinct histological subtypes; tubular, papillary, mucinous and signet-ring cell carcinomas ${ }^{(10)}$. The Laurén classification categorizes GC tumors into intestinal- and diffuse-types, and is useful for correlating the histological appearance of tumors with disease natural history, such as its association with environmental factors, precursor lesions, and incidence trends ${ }^{(10)}$. Tumors that display similar distributions of intestinal- and diffuse-type histology are described as mixed, and undifferentiated tumors are classified as indeterminate.

Intestinal-type tumors account for the vast majority of sporadic GC and are associated with better prognosis compared to the diffuse-type. They form poorly differentiated glands, usually in conjunction with intestinal metaplasia, whereas the diffuse-type consists of small cohesive cells, with diffuse infiltration in the organ wall and poor or absent glandular differentiation ${ }^{(10)}$. Intestinal-type tumors are strongly associated with defined risk factors; for example, its occurrence is closely related to the presence of Helicobacter pylori infection, which can lead to atrophic gastritis, followed by intestinal metaplasia and neoplastic transformation. This type of GC is also associated with obesity and gastroesophageal reflux disease, as well as age and diet.

Diffuse-type GC is not clearly related to defined environmental risk factors, but is associated with $\mathrm{CDH} 1$ (E-cadherin) mutation and is the histological type most typical of genetic syndromes associated with GC. The relative incidence of Laurén histological types varies according to the population studied $^{(10)}$.

\section{Epstein-Barr virus in gastric cancer}

Approximately $90 \%$ of the world's population is infected by $\mathrm{EBV}^{(19)}$; however, only a minority of infected individuals develop EBV-associated malignancies, including GC. The reasons that neoplastic transformation occurs in a small number of patients, while the majority are spared, are unknown. 
In healthy individuals, infection of the gastric epithelial cells is rare ${ }^{(20)(21)(22)}$, and the mechanisms underlying infection of the gastric mucosa by EBV remain unknown. It is assumed that EBV infection is an early event in carcinogenesis, owing to evidence of virus infection in premalignant lesions, such as dysplastic epithelial cells ${ }^{(2)(21)(23)}$.

EBV-positive GC is defined by the presence of virus in tumor cells. It tends to present with a distinct clinicopathological phenotype, compared to EBV-negative GC. Meta-analysis involving 9,738 patients from 48 studies revealed a rate of infection of $8.8 \% \%^{(19)}$. EBV-positive GC was more prevalent in younger patients (50-68 years old), compared to EBV-negative tumors (56-72 years-old) ${ }^{(19)(24)}$, and is associated with male sex, as well as Caucasian and Hispanic ethnicities ${ }^{(19)(24)(25)(26)(27) .}$ EBV-positive tumors occur preferentially in proximal portions of the stomach, most frequently the cardia and gastric body ${ }^{(19)}$ (24) (26), and are associated with diffuse-type histology ${ }^{(19)(24)}$.

There is a strong relationship between EBV infection and lymphoepithelioma-like gastric carcinoma, as well as gastric remnant carcinomas, particularly those that undergo Billroth II surgery, suggesting that damage to the gastric mucosa facilitates EBV infection in the remaining tissues ${ }^{(19)}$.

The association of EBV infection and tumor depth is controversial. Studies have shown correlation with both superficial and more invasive tumors. An association with nodal status is also debated. A study involving 715 patients demonstrated that EBV-positive GC patients tend to present without nodal involvement ${ }^{(18)}$, whereas another study of 235 patients revealed an association between viral infection and the presence of nodal metastases ${ }^{(23)}$. Meta-analysis showed no correlation between EBV infection and tumor depth or nodal status $^{(19)}$.

\section{Genetic and epigenetic abnormalities}

EBV-positive GC patients have typical genetic and epigenetic alterations, which translate into a clinicopathological phenotype defining a specific subtype of GC.

Nine well-recognized viral genes (BARF0, BARF1, BcLF1, BHRF1, BLLF1, BRLF1, BZLF1, EBNA1, and LMP2A) are highly expressed in EBV-positive $\mathrm{GC}^{(28)(29)(30)(31)}$. Some of these genes, including BARF1, BHRF1, and LMP2A, have oncogenic potential ${ }^{(28)}$. Expression of LMP2A is involved in up-regulation of survivin protein, which confers a cell survival advantage, and activates cellular DNMT3b, causing genome-wide aberrant methylation in host cells ${ }^{(28)(32)}$.

EBV exists as episomes in host cell nuclei, owing to the length of its genome (approximately 170kb) ${ }^{(2)}(28)$, and approximately 205 host cell genes are typically mutated in EBV-positive GC, including AKT2, CCNA1, MAP3K4 and $T G F B R l^{(28)}$.

$A K T 2$ is a putative oncogene encoding a protein that participates in important cancer pathways, including MAPK signaling. The mutant form of $A K T 2$ identified in EBV-positive GC exhibits elevated kinase activity, with consequent increases in the activities of the important mediators of the MAPK signaling pathway, AP-1 and ERK, leading to promotion of cell growth. Cyclin A1 (CCNA1) belongs to the cyclin family and functions in the control of the germline meiotic cell cycle. CCNA1 plays different roles in virus-related and non-virusrelated malignancies. MAP3K4 functions as a major mediator of environmental stressors that activate the p38 MAPK pathway, and its mutation has been reported in endometrial cancer. Transforming growth factor- $\beta$-receptor 1 (TGFBR1) is a serine/threonine protein kinase and receptor for TGF- $\beta$. Mutations in TGFBR1 have been found in skin and colorectal cancers. Given the functional importance of these genes in human cancers, their EBV-induced mutation may contribute to the pathogenesis of EBV-associated $\mathrm{GC}^{(28)}$.

Virus-related epigenetic alterations are also apparent in EBV host cells. Studies of cultured EBV-positive GC cells demonstrated that 216 genes were hypermethylated and transcriptionally down-regulated, and 46 were demethylated and transcriptionally up-regulated, compared to their expression in EBV-negative cells ${ }^{(28)(33)}$. Similar findings were reported in tumor samples, with high levels of methylation of the $A C S S 1, F A M 3 B$, $I H H$, and TRABD genes $^{(28)}$. Knockdown of IHH and TRABD induces an increase in tumor cell proliferation, demonstrating that such genes have potential tumor-suppressor functions; hence, their methylation could be involved in the pathogenesis of EBV-positive $\mathrm{GC}^{(28)}$. There is evidence for an association between EBV-positive GC and CpG methylation ${ }^{(32)(34)(35)}$. The $\mathrm{CpG}$ island methylator phenotype (CIMP) is characterized by simultaneous methylation of multiple genes and is an important mechanism of gastrointestinal tumor carcinogenesis. Furthermore, CIMP status (CIMP-high/CIMP-low or CIMPnegative) appears to correlate with prognosis. CIMP-high patients tend to have improved prognosis, more superficial tumors, diffuse-type histology, and early-stage disease, whereas CIMP-negative patients generally have poorer prognosis ${ }^{(35)}(36)$. This suggests that EBV infection causes hypermethylation of a specific group of genes, and that silencing of these genes may favor the malignant transformation of gastric epithelial cells.

Genetic and epigenetic abnormalities identified in EBVpositive GC comprise five interrelated core pathways, namely axon guidance, focal adhesion, cytokine-cytokine receptor interaction, MAPK signaling, and regulation of actin cytoskeleton. Three of these pathways (cytokine-cytokine receptor interaction, MAPK signaling, and regulation of actin cytoskeleton) are affected by EBV infection in lymphoblastoid cell lines and primary B cells, suggesting common dysregulation of these pathways by EBV infection in different cell types during disease initiation. Dysregulation of these five core pathways, through both genetic and epigenetic modulation of host genes by EBV infection, may be important in the development of this subtype of $\mathrm{GC}^{(28)}$.

The recent TCGA molecular classification divides GC into four subtypes: EBV-positive, microsatellite unstable (MSI), genomically stable (GS), and chromosomal instability (CIN), based on data produced using six molecular platforms (arraybased somatic copy number analysis, whole-exome sequencing, array-based DNA methylation profiling, messenger RNA sequencing, microRNA sequencing, and reverse-phase protein array) $^{(15)}$ (Figure 2). 


\section{EBV}

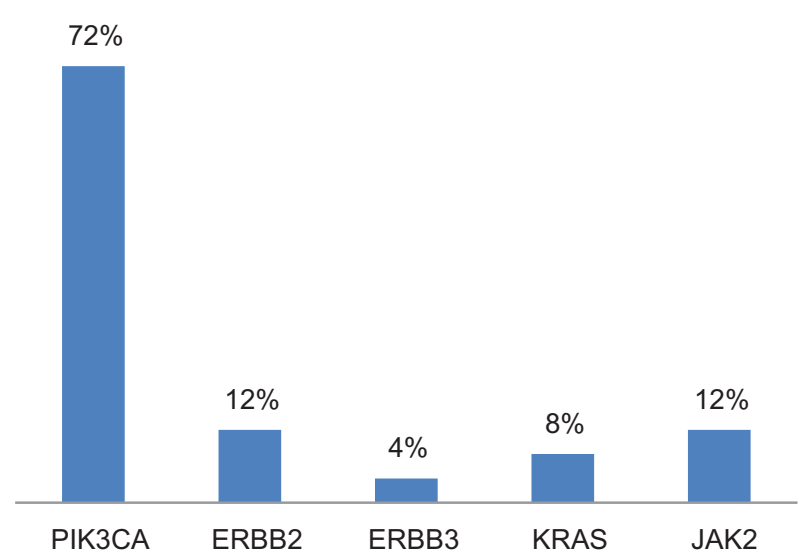

GS



MSI
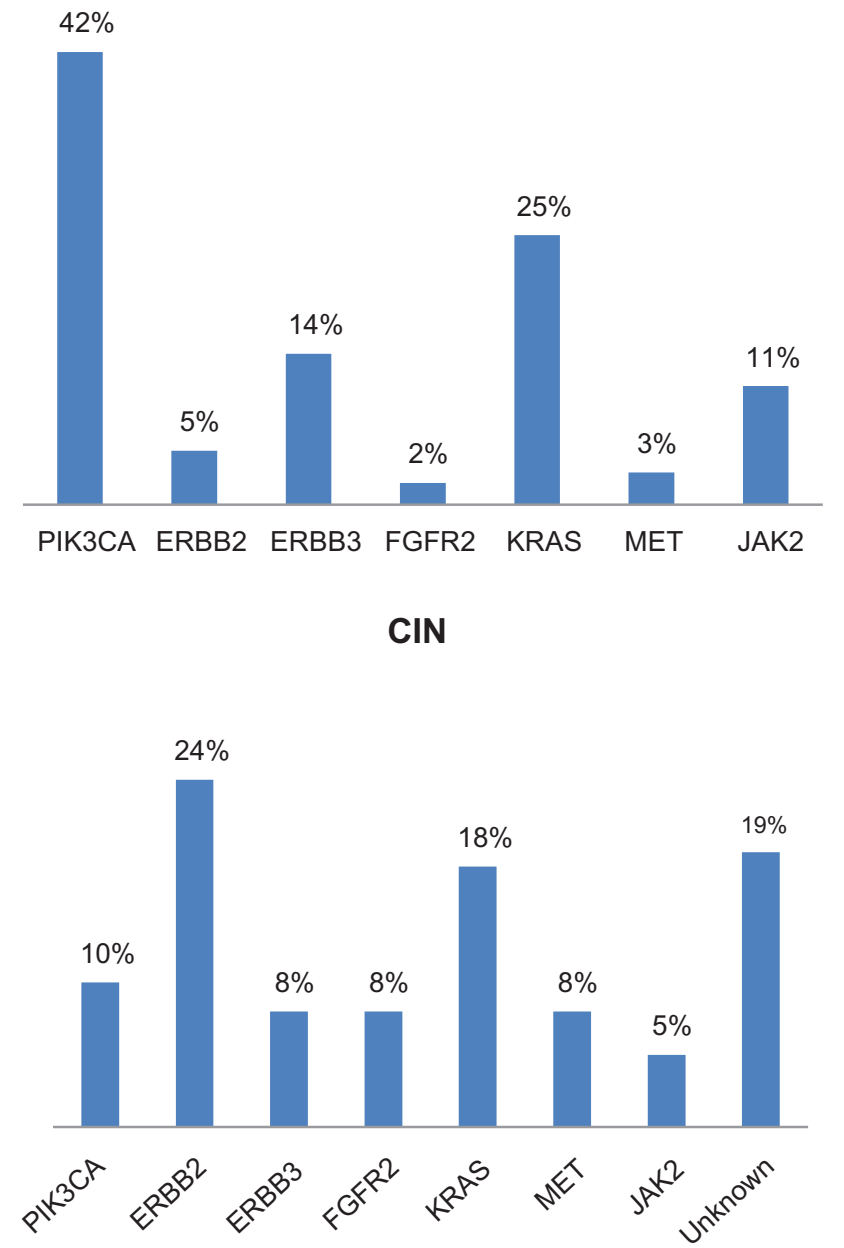

FIGURE 2 - Proportion of genetic abnormalities in distinct GC subtypes. EBV: Epstein-Barr virus; MSI: microsatellite-instability; GS: genomically stable; CIN: Chromosomal instability.

Nine per cent of tumors in the TCGA study were assigned to the EBV-positive subtype, which is associated with the most extensive DNA hypermethylation identified in solid tumors by TCGA to date ${ }^{(15)}$. EBV-positive subtype tumors are predominantly located in the gastric fundus or body, and preferentially occur in males. ARIDIA and BCOR mutations are prevalent, whereas genetic alterations of TP53 are rare ${ }^{(15)(37)}$. Notably, $80 \%$ of EBVpositive subtype tumors harbor mutations in phosphatidylinositol 3-kinase CA (PIK3CA) and amplification of $J A K 2, C D 274$, and $P D C D 1 L G 2$, which encode receptor tyrosine kinase, PD-L1 and PD-L2, respectively, are also common ${ }^{(15)}$. Based on these findings, JAK2 inhibitors and PD-L1/2 antagonists could be explored as treatment options in EBV-positive tumors.

In contrast, MSI-tumors tend to occur more frequently in females and older patients. These represent $22 \%$ of cases and are strongly correlated with $M L H 1$ hypermethylation. GStumors are associated with diffuse-type histology and tend to be diagnosed at an earlier age. $\mathrm{CDH} 1$ mutations are enriched in this subgroup, which represents $20 \%$ of GC patients. As in the EBV-subtype, ARID1A mutations are prevalent, and mutations of the RHOA gene are almost exclusively found in GS tumors. The CIN-subtype represents the largest group, accounting for $50 \%$ of cases. It has a predilection for the gastroesophageal junction, an association with intestinal-type histology, and the highest rates of ERBB2 amplification among the molecular subtypes. Elevated rates of EGFR amplification and TP53 mutation are also characteristic of the CIN-subtype, consistent with the marked aneuploidy also distinguishing this group.

There was no difference in survival among patients with the four molecular subtypes evaluated in the TCGA study and comparison of the distribution of subtypes between western and eastern patients revealed a similar distribution in both populations ${ }^{(15)}$. Additional studies including larger numbers of patient samples are needed to better clarify the relationships among geographic regions, ethnic characteristics, and the biological profile of GC. 


\section{Prognostic value of EBV}

The impact of EBV infection on the overall survival of GC patients remains a topic of debate. All studies that have evaluated this issue were retrospective and, therefore, provide an unsatisfactory level of evidence to allow definitive conclusions.

An international pooled analysis including 13 studies, totaling 4,599 patients, suggested that EBV-positive GC is associated with better prognosis, with a $28 \%$ reduction in relative risk of death (HR, 0.72; 95\% CI, 0.61-0.86) ${ }^{(38)}$. EBVpositive patients had an estimated median survival of 8.5 years compared to 5.3 years for EBV-negative patients $(\mathrm{p}=0.006)$. EBV infection status was a prognostic factor, alongside TNM stage, age, anatomic subsite, and degree of differentiation. Likewise, exploratory analysis of a pivotal Dutch Trial, investigating the prognostic influence of EBV status, showed better cancer-related survival and disease-free survival in an EBV-positive patient subgroup, which could be explained by decreased nodal involvement, reduced residual disease and younger age in this subgroup ${ }^{(18)}$.

By contrast, in a retrospective analysis including $123 \mathrm{EBV}$ positive GC patients and $405 \mathrm{EBV}$-negative controls, superior overall survival was demonstrated in the former group, but only in univariate analysis. The statistical significance was lost when other prognostic variables were included in multivariate analysis $^{(39)}$. Similarly, a study where 457 patients samples were analyzed by tissue microarray did not identify any correlation between EBV infection and survival ${ }^{(27)}$.

Host immune response to EBV infection appears to influence the prognosis of neoplastic disease, mainly when predominated by CD3+ and CD8+ T-lymphocytes, and is one hypothesis to explain the distinct pattern of survival in this subtype of GC. In lymphoepithelioma-like carcinoma, a rare GC and an established EBV-associated form of the disease, three histological subtypes can be identified based on host inflammatory response, typical lymphoepithelioma-like carcinoma, Crohn's disease-like lymphocytic reaction, and conventional adenocarcinoma. The first two subtypes appear to be associated with better $\operatorname{prognosis}^{(39)}$.

\section{EBV-positive GC in Brazil}

EBV-positive GC patients from the Brazilian population appear to present with similar prevalence and clinicopathological features to those reported worldwide. A study of 149 Brazilians of Japanese origin and 151 without Japanese origin demonstrated $4.7 \%$ and $11.2 \%$ of EBV-positive GC in each group, respectively ${ }^{(40)}$. Another study involving 53 Brazilian patients from the State of São Paulo revealed $11.3 \%$ positivity, with male predominance $(83.3 \%)$, median age of 59 years and a higher incidence of lesions in the gastric antrum $(41.5 \%)^{(41)}$. Similarly, a prevalence of $9.6 \%$ was found in 39 patients from the City of Belém, State of Pará ${ }^{(20)}$, and another study, also involving a population from Northern Brazil, but including only ten GC patients and six controls without tumor, found that eight patients had EBV DNA in their tumor cells, with no positivity in the control group ${ }^{(22)}$. There is no data available about the prognostic value of EBV infection in the Brazilian population.

\section{Therapeutic implications}

The identification of genetic and epigenetic abnormalities and the recognition of the complex relationships between them are the first steps towards genome-guided personalized therapy. In recent years, several genetic and epigenetic abnormalities have been demonstrated in GC, leading to the identification of potential therapeutic targets (Figure 1). These results have emerged from exome sequencing studies of small numbers of patient samples; therefore, the global molecular portrait of GC remains incomplete. Nevertheless, several genetic abnormalities have been described, and some have been further validated, demonstrated to be targetable, and have already resulted in clinical studies or promising ongoing trials (Table 1). Furthermore, the rates of these abnormalities seem to differ according to GC-molecular subtype ${ }^{(15)}$ (Figure 2). In respect of genetic and epigenetic abnormalities enriched in EBVpositive GC, the pathways detailed in the following sections have been explored for therapeutic potential.

\section{PIK3CA/Akt pathway}

As mentioned above, $80 \%$ of EBV-positive GC patients have PIK3CA mutations. Phosphatidylinositol 3-kinases (PIK3)

TABLE 1 - Targeted therapies in advanced gastric cancer potentially applicable to Epstein-Barr virus positive patients.

\begin{tabular}{lccc}
\hline Target & Drug & Study phase & Status \\
\hline PIK3/Akt/mTOR & Everolimus & Phase III & Available results[47] \\
& & Phase III & Ongoing (NCT01248403) \\
& & Phase I & Ongoing (NCT01049620) \\
& MK-2206 & Phgoing (NCT01260701) & Ongoing (NCT01613950) \\
& BYL719 & Phase I & Available results[55] \\
PD-L1 & Pembrolizumab & Phase Ib & Ongoing (NCT02335411) \\
& & Phase II & Ongoing (NCT02340975) \\
CTLA-4 & & Phase I/II & Ongoing (NCT01219543) \\
JAK2 & Tremelimumab & Phase I & AZD1480
\end{tabular}


are heterodimeric lipid kinases composed of several regulatory subunits. In response to stimulation by growth factors, PIK3CA, which encodes the p110 alpha catalytic subunit of PIK3, induces activation of downstream effectors, including pAkt and $\mathrm{mTOR}^{(42)}$. PIK3CA amplification contributes to cell proliferation and survival in gastric tumorigenesis, through the activation of the PIK3/pAkt pathway ${ }^{(43)}$. PIK3CA mutations have been detected at various frequencies (4-15\%) in populations of GC patients ${ }^{(44)(45)(46)}$; however, its distribution varies widely among the molecular subtypes, with the highest incidence in the EBV subtype $(80 \%)$ and the lowest in the CIN subtype $(3 \%)^{(15)}$.

There are no clinical trials with available results evaluating PIK3/pAkt pathway inhibitors in GC. Phase II clinical trials, investigating Akt inhibitors, such as MK-2206, in secondline treatment of advanced GC, are ongoing (clinicaltrials. govNCT01260701). As PIK3 mutations can induce resistance to HER2 inhibition, MK-2206 is also being tested in association with lapatinib and trastuzumab in HER2-positive GC patients (clinicaltrials.govNCT01705340). BYL719, another PIK3 inhibitor, is under evaluation, in a phase I trial, in association with AUY922, a HSP90 inhibitor, in patients who harbor molecular alteration of $P I K 3$ or amplification of ERBB2 (clinicaltrials.govNCT01613950).

The PIK3/Akt/mTOR pathway is up-regulated in several solid tumors, and estimated to be activated in up to $60 \%$ of GC patients, through PTEN loss of function or PIK3CA-activating mutations. Everolimus, a well-known mTOR inhibitor, was studied in patients with advanced $\mathrm{GC}$ who failed standard therapies, in a placebo-controlled, phase III trial (GRANITE-1 study) $)^{(47)}$. The study failed to show the advantages of everolimus over best supportive care in overall survival (5.4 months vs. 4.3 months; HR, $0.90 ; 95 \%$ CI, $0.75-1.08)$. Biomarkers that could predict benefit of everolimus treatment have been elusive and the results of biomarker analysis from the GRANITE-1 trial are awaited. Despite initial disappointing results, strategies forPI3K/Akt/mTOR pathway inhibition are currently under investigation, with everolimus in combination with capecitabine and oxaliplatin in a phase I study (clinicaltrials.govNCT01049620), and with paclitaxel in a phase III trial in second-line setting (clinicaltrials.govNCT01248403).

\section{PD-1/PD-L1, PD-L2 pathway}

The blockade of immune checkpoints is one of the most promising approaches to activating therapeutic antitumor immunity. The best-described immune checkpoints are the CTLA-4 and PD-1 receptors, which are linked to immune inhibitory pathways, and are crucial for maintaining selftolerance and modulating physiological immune responses to minimize collateral tissue damage ${ }^{(48)}$. PD-1 has two ligands, PD-L1 and PD-L2. Therapeutic strategies to block the CTLA4 and PD-1 pathways, both alone and in combination, have been successful in metastatic melanoma ${ }^{(49)(50)(51)}$, and present a promising therapeutic target in other malignant neoplasms.

Expression of PD-L1 and PD-L2 is elevated in EBVpositive $\mathrm{GC}^{(15)}$. In an unselected GC population, PD-L1 was overexpressed in $42 \%-50 \%$ of patients ${ }^{(52)(53)}$, and was correlated with poor survival rates ${ }^{(53)}$. Inhibition of PD-L1 in GC using monoclonal antibodies has been explored in experimental models ${ }^{(54)}$, and evaluated in a phase $\mathrm{Ib}$ trial, which demonstrated antitumor activity of pembrolizumab in an enriched PD-L1-positive population composed of $39 \mathrm{GC}$ patients ${ }^{(5)}$. Pembrolizumab is also currently being tested in a phase II study evaluating its efficacy in previously treated HER2-negative advanced GC (clinicaltrials.govNCT02335411). Inhibition of CTLA-4 is also being investigated in GC, with the use of tremelimumab in a phase $\mathrm{Ib} / \mathrm{II}$ trial in patients with refractory disease (clinicaltrials.govNCT02340975).

\section{JAK2 pathway}

The Janus kinase (JAK) family of proteins is composed of non-receptor tyrosine kinases, which phosphorylate cytoplasmic targets, including the signal transducers and activators of transcription (STATs) ${ }^{(56)}$. The JAK/STAT pathway mediates signaling through cytokines, and is required for cell proliferation, survival, and differentiation. JAK2 is a member of this family, and its mutation is a well-known genetic alteration in myeloproliferative disorders ${ }^{(57)}$. In GC, JAK2 overexpression appears to be enriched in the EBV-molecular subtype ${ }^{(15)}$. Inhibition of the JAK2/STAT3 pathway with WP1066 reduced GC growth in experimental models, and may form the basis for further clinical studies ${ }^{(58)}$. Clinical use of a JAK2 inhibitor, AZD1480, is being evaluated in a phase I, dose-escalation study in Asian patients with solid malignancies, including GC (clinicaltrials.govNCT01219543).

In conclusion, EBV-positive GC is the largest group of EBV-associated malignancies. Previous studies have identified a distinct clinicopathological phenotype of EBV-positive $\mathrm{GC}$, demonstrating that this subgroup of patients tend to be younger, predominantly male, and to have diffuse-type histology tumors in proximal portions of the stomach, and better overall survival. Gene expression profile studies have provided detailed molecular characterization of GC, confirming that EBV-positive $\mathrm{GC}$ is a distinct molecular subtype of the disease, with unique genetic and epigenetic abnormalities.

These findings highlight the heterogeneity of GC, and the complex interplay between genetic and epigenetic alterations. The identification of these characteristic molecular alterations provides potential novel therapeutic targets and a roadmap to implement genome-guided personalized therapy in gastric cancer.

\section{CONFLICT OF INTEREST}

The authors declare that there is no conflict of interest.

\section{FINANCIAL SUPPORT}

Supported by FundaçãoWaldemar Barnsley Pessoa, Ribeirão Preto, SP, Brazil.

\section{REFERENCES}

1. Epstein MA, Achong BG, Barr YM. Virus particles in cultured lymphoblasts from Burkitt's lymphoma. Lancet 1964; 1:702-703. 
2. Young LS, Rickinson AB. Epstein-Barr virus: 40 years on. Nat Rev Cancer 2004; 4:757-768.

3. Torre LA, Bray F, Siegel RL, Ferlay J, Lortet-Tieulent J, Jemal A. Global cancer statistics, 2012. CA Cancer J Clin 2015; 65:87-108.

4. Takada K. Epstein-Barr virus and gastric carcinoma. Mol Pathol 2000; 53:255-261.

5. Sousa H, Pinto-Correia AL, Medeiros R, Dinis-Ribeiro M. Epstein-Barr virus is associated with gastric carcinoma: the question is what is the significance? World J Gastroenterol 2008; 14:4347-4351.

6. Burke AP, Yen TS, Shekitka KM, Sobin LH. Lymphoepithelial carcinoma of the stomach with Epstein-Barr virus demonstrated by polymerase chain reaction. Mod Pathol 1990; 3:377-380.

7. Shibata D, Tokunaga M, Uemura Y, Sato E, Tanaka S, Weiss LM. Association of Epstein-Barr virus with undifferentiated gastric carcinomas with intense lymphoid infiltration. Lymphoepitheliomalike carcinoma. Am J Pathol 1991; 139:469-474.

8. Yamamoto N, Tokunaga M, Uemura Y, Tanaka S, Shirahama H, Nakamura T, et al. Epstein-Barr virus and gastric remnant cancer. Cancer 1994; 74:805-809.

9. Baas IO, van Rees BP, Musler A, Craanen ME, Tytgat GN, van den Berg FM, et al. Helicobacter pylori and Epstein-Barr virus infection and the p53 tumour suppressor pathway in gastric stump cancer compared with carcinoma in the non-operated stomach. J Clin Pathol 1998; 51:662-666.

10. Bosman FT, Carneiro F, Hruban RH, Theise ND. WHO Classificaton of Tumors of the Digestive System. $4^{\text {th }}$ edition. In: Bosman FT, Carneiro F, Hruban RH, Theise ND (editors). WHO Classificaton of Tumors, volume 3. Lyon, France: IARC Press; 2010. 417p.

11. Ajani JA, Rodriguez W, Bodoky G, Moiseyenko V, Lichinitser $\mathrm{M}$, Gorbunova V, et al. Multicenter phase III comparison of cisplatin/S-1 with cisplatin/infusional fluorouracil in advanced gastric or gastroesophageal adenocarcinoma study: the FLAGS trial. J Clin Oncol 2010; 28:1547-1553.

12. Ohtsu A, Shah MA, Van Cutsem E, Rha SY, Sawaki A, Park SR, et al. Bevacizumab in combination with chemotherapy as first-line therapy in advanced gastric cancer: a randomized, double-blind, placebo-controlled phase III study. J Clin Oncol 2011; 29:3968-3976.

13. Bang YJ, Van Cutsem E, Feyereislova A, Chung HC, Shen L, Sawaki A, et al. Trastuzumab in combination with chemotherapy versus chemotherapy alone for treatment of HER2-positive advanced gastric or gastro-oesophageal junction cancer (ToGA): a phase 3, open-label, randomised controlled trial. Lancet 2010; 376:687-697.

14. Jácome AA, Wohnrath DR, Scapulatempo Neto C, Carneseca EC, Serrano SV, Viana LS, et al. Prognostic value of epidermal growth factor receptors in gastric cancer: a survival analysis by Weibull model incorporating long-term survivors. Gastric Cancer 2014; 17:76-86.

15. Network CGAR. Comprehensive molecular characterization of gastric adenocarcinoma. Nature 2014; 513:202-209.

16. Wu MS, Shun CT, Wu CC, Hsu TY, Lin MT, Chang MC, et al. Epstein-Barr virus-associated gastric carcinomas: relation to H. pylori infection and genetic alterations. Gastroenterol 2000; 118:1031-1038.

17. zur Hausen A, van Grieken NC, Meijer GA, Hermsen MA, Bloemena E, Meuwissen SG, et al. Distinct chromosomal aberrations in Epstein-Barr virus-carrying gastric carcinomas tested by comparative genomic hybridization. Gastroenterol 2001; 121:612-618.
18. van Beek J, zur Hausen A, Klein Kranenbarg E, van de Velde CJ, Middeldorp JM, van den Brule AJ, et al. EBV-positive gastric adenocarcinomas: a distinct clinicopathologic entity with a low frequency of lymph node involvement. J Clin Oncol 2004; 22:664-670.

19. Lee JH, Kim SH, Han SH, An JS, Lee ES, Kim YS. Clinicopathological and molecular characteristics of Epstein-Barr virus-associated gastric carcinoma: a meta-analysis. J Gastroenterol Hepatol 2009; 24:354-365.

20. Liang Q, Yao X, Tang S, Zhang J, Yau TO, Li X, et al. Integrative identification of Epstein-Barr virus-associated mutations and epigenetic alterations in gastric cancer. Gastroenterol 2014; 147:1350-1362.e4.

21. de Souza CR, de Oliveira KS, Ferraz JJ, Leal MF, Calcagno DQ, Seabra AD, et al. Occurrence of Helicobacter pylori and EpsteinBarr virus infection in endoscopic and gastric cancer patients from Northern Brazil. BMC Gastroenterol 2014; 14:179.

22. Martínez-López JL, Torres J, Camorlinga-Ponce M, Mantilla A, Leal YA, Fuentes-Pananá EM. Evidence of Epstein-Barr virus association with gastric cancer and non-atrophic gastritis. Viruses 2014; 6:301-318.

23. de Aquino PF, Carvalho PC, da Gama Fischer JS, de Souza AQ, Viana JS, Chalub SR, et al. Epstein-Barr virus DNA associated with gastric adenocarcinoma and adjacent non-cancerous mucosa in patients from Manaus, Brazil. Genet Mol Res 2012; 11:4442-4446.

24. Truong CD, Feng W, Li W, Khoury T, Li Q, Alrawi S, et al. Characteristics of Epstein-Barr virus-associated gastric cancer: a study of 235 cases at a comprehensive cancer center in U.S.A. J Exp Clin Cancer Res 2009; 28:14.

25. Luo B, Wang Y, Wang XF, Liang H, Yan LP, Huang BH, et al. Expression of Epstein-Barr virus genes in EBV-associated gastric carcinomas. World J Gastroenterol 2005; 11:629-633.

26. Sivachandran N, Dawson CW, Young LS, Liu FF, Middeldorp J, Frappier L. Contributions of the Epstein-Barr virus EBNA1 protein to gastric carcinoma. J Virol 2012; 86:60-68.

27. Chang MS, Kim DH, Roh JK, Middeldorp JM, Kim YS, Kim S, et al. Epstein-Barr virus-encoded BARF1 promotes proliferation of gastric carcinoma cells through regulation of NF-אB. J Virol 2013; 87:10515-10523.

28. Zhao J, Liang Q, Cheung KF, Kang W, Lung RW, Tong JH, et al. Genome-wide identification of Epstein-Barr virus-driven promoter methylation profiles of human genes in gastric cancer cells. Cancer 2013; 119:304-312.

29. Sudo M, Chong JM, Sakuma K, Ushiku T, Uozaki H, Nagai H, et al. Promoter hypermethylation of E-cadherin and its abnormal expression in Epstein-Barr virus-associated gastric carcinoma. Int J Cancer 2004; 109:194-199.

30. Kim J, Lee HS, Bae SI, Lee YM, Kim WH. Silencing and CpG island methylation of GSTP1 is rare in ordinary gastric carcinomas but common in Epstein-Barr virus-associated gastric carcinomas. Anticancer Res 2005; 25:4013-4019.

31. Kusano M, Toyota M, Suzuki H, Akino K, Aoki F, Fujita M, et al. Genetic, epigenetic, and clinicopathologic features of gastric carcinomas with the $\mathrm{CpG}$ island methylator phenotype and an association with Epstein-Barr virus. Cancer 2006; 106:1467-1479.

32. Chang MS, Uozaki H, Chong JM, Ushiku T, Sakuma K, Ishikawa $\mathrm{S}$, et al. $\mathrm{CpG}$ island methylation status in gastric carcinoma with and without infection of Epstein-Barr virus. Clin Cancer Res 2006; 12:2995-3002.

33. Abe H, Maeda D, Hino R, Otake Y, Isogai M, Ushiku AS, et al. ARID1A expression loss in gastric cancer: pathway-dependent roles with and without Epstein-Barr virus infection and microsatellite instability. Virchows Arch 2012; 461:367-377. 
34. Camargo MC, Murphy G, Koriyama C, Pfeiffer RM, Kim WH, Herrera-Goepfert R, et al. Determinants of Epstein-Barr viruspositive gastric cancer: an international pooled analysis. $\mathrm{Br} \mathrm{J}$ Cancer 2011; 105:38-43.

35. Vo QN, Geradts J, Gulley ML, Boudreau DA, Bravo JC, Schneider BG. Epstein-Barr virus in gastric adenocarcinomas: association with ethnicity and CDKN2A promoter methylation. J Clin Pathol 2002; 55:669-675.

36. Murphy G, Pfeiffer R, Camargo MC, Rabkin CS. Meta-analysis shows that prevalence of Epstein-Barr virus-positive gastric cancer differs based on sex and anatomic location. Gastroenterol 2009; 137:824-833.

37. Huang SC, Ng KF, Chen KH, Hsu JT, Liu KH, Yeh TS, et al. Prognostic factors in Epstein-Barr virus-associated stage I-III gastric carcinoma: implications for a unique type of carcinogenesis. Oncol Rep 2014; 32:530-538.

38. Camargo MC, Kim WH, Chiaravalli AM, Kim KM, Corvalan AH, Matsuo K, et al. Improved survival of gastric cancer with tumour Epstein-Barr virus positivity: an international pooled analysis. Gut 2014; 63:236-243.

39. Song HJ, Srivastava A, Lee J, Kim YS, Kim KM, Ki Kang W, et al. Host inflammatory response predicts survival of patients with Epstein-Barr virus-associated gastric carcinoma. Gastroenterol 2010; 139:84-92.e2.

40. Koriyama C, Akiba S, Iriya K, Yamaguti T, Hamada GS, Itoh T, et al. Epstein-Barr virus-associated gastric carcinoma in Japanese Brazilians and non-Japanese Brazilians in São Paulo. Jpn J Cancer Res 2001; 92:911-917.

41. Lopes LF, Bacchi MM, Elgui-de-Oliveira D, Zanati SG, Alvarenga $\mathrm{M}$, Bacchi CE. Epstein-Barr virus infection and gastric carcinoma in São Paulo State, Brazil. Braz J Med Biol Res 2004; 37:1707-1712.

42. Jia S, Liu Z, Zhang S, Liu P, Zhang L, Lee SH, et al. Essential roles of $\mathrm{PI}(3) \mathrm{K}$-p110beta in cell growth, metabolism and tumorigenesis. Nature 2008; 454:776-779.

43. Byun DS, Cho K, Ryu BK, Lee MG, Park JI, Chae KS, et al. Frequent monoallelic deletion of PTEN and its reciprocal associatioin with PIK3CA amplification in gastric carcinoma. Int J Cancer 2003; 104:318-327.

44. Li VS, Wong CW, Chan TL, Chan AS, Zhao W, Chu KM, et al. Mutations of PIK3CA in gastric adenocarcinoma. BMC Cancer 2005; 5:29.

45. Velho S, Oliveira C, Ferreira A, Ferreira AC, Suriano G, Schwartz $\mathrm{S}$, et al. The prevalence of PIK3CA mutations in gastric and colon cancer. Eur J Cancer 2005; 41:1649-1654.
46. Zhou J, Chen GB, Tang YC, Sinha RA, Wu Y, Yap CS, et al. Genetic and bioinformatic analyses of the expression and function of PI3K regulatory subunit PIK3R3 in an Asian patient gastric cancer library. BMC Med Genomics 2012; 5:34.

47. Ohtsu A, Ajani JA, Bai YX, Bang YJ, Chung HC, Pan HM, et al. Everolimus for previously treated advanced gastric cancer: results of the randomized, double-blind, phase III GRANITE-1 study. J Clin Oncol 2013; 31:3935-3943.

48. Pardoll DM. The blockade of immune checkpoints in cancer immunotherapy. Nat Rev Cancer 2012; 12:252-264.

49. Hodi FS, O'Day SJ, McDermott DF, Weber RW, Sosman JA, Haanen JB, et al. Improved survival with ipilimumab in patients with metastatic melanoma. N Engl J Med 2010; 363:711-723.

50. Hamid O, Robert C, Daud A, Hodi FS, Hwu WJ, Kefford R, et al. Safety and tumor responses with lambrolizumab (anti-PD-1) in melanoma. N Engl J Med 2013; 369:134-144.

51. Robert C, Long GV, Brady B, Dutriaux C, Maio M, Mortier L, et al. Nivolumab in previously untreated melanoma without BRAF mutation. N Engl J Med 2015; 372:320-330.

52. Qing Y, Li Q, Ren T, Xia W, Peng Y, Liu GL, et al. Upregulation of PD-L1 and APE1 is associated with tumorigenesis and poor prognosis of gastric cancer. Drug Des Devel Ther 2015; 9:901-919.

53. WuC, Zhu Y, Jiang J,Zhao J,Zhang XG, Xu N. Immunohistochemical localization of programmed death-1 ligand-1 (PD-L1) in gastric carcinoma and its clinical significance. Acta Histochem 2006; 108:19-24.

54. Sun J, Xu K, Wu C, Wang Y, Hu Y, Zhu Y, et al. PD-L1 expression analysis in gastric carcinoma tissue and blocking of tumorassociated PD-L1 signaling by two functional monoclonal antibodies. Tissue Antigens 2007; 69:19-27.

55. Muro K, Bang Y, Shankaran V, Geva R, Catenacci D, Gupta S, et al. A phase Ib study of pembrolizumab (Pembro; MK-3475) in patients (Pts) with advanced gastric cancer. Ann Oncol 2014; 25:141.

56. Kralovics R, Passamonti F, Buser AS, Teo SS, Tiedt R, Passweg JR, et al. A gain-of-function mutation of JAK2 in myeloproliferative disorders. N Engl J Med 2005; 352:1779-1790.

57. Levine RL, Pardanani A, Tefferi A, Gilliland DG. Role of JAK2 in the pathogenesis and therapy of myeloproliferative disorders. Nat Rev Cancer 2007; 7:673-683.

58. Judd LM, Menheniott TR, Ling H, Jackson CB, Howlett M, Kalantzis A, et al. Inhibition of the JAK2/STAT3 pathway reduces gastric cancer growth in vitro and in vivo. PLoS One 2014; 9:e95993. 